Relations industrielles

Industrial Relations

\title{
Une analyse des gains au Canada, par Peter Kuch et Walter Haessel, Ottawa, Statistique Canada, 1979, 272 pp.
}

\section{Gérard Marion}

Volume 34, numéro 3, 1979

URI : https://id.erudit.org/iderudit/028997ar

DOI : https://doi.org/10.7202/028997ar

Aller au sommaire du numéro

Éditeur(s)

Département des relations industrielles de l'Université Laval

ISSN

0034-379X (imprimé)

1703-8138 (numérique)

Découvrir la revue

Citer ce compte rendu

Marion, G. (1979). Compte rendu de [Une analyse des gains au Canada, par Peter Kuch et Walter Haessel, Ottawa, Statistique Canada, 1979, 272 pp.] Relations industrielles / Industrial Relations, 34(3), 617-619.

https://doi.org/10.7202/028997ar

Tous droits réservés @ C Département des relations industrielles de l'Université Laval, 1979
Ce document est protégé par la loi sur le droit d'auteur. L'utilisation des services d'Érudit (y compris la reproduction) est assujettie à sa politique d'utilisation que vous pouvez consulter en ligne.

https://apropos.erudit.org/fr/usagers/politique-dutilisation/ 
German experience mentions, among others, that effective management has not been sacrificed to any significant extent. "So far at least, management has been left with a relatively free hand to operate efficiently, provided it allows for and takes into consideration the effects on workers of literally everything it decides and does. Assuming due allowance is made for their concerns, worker representatives on company boards and works councils do not tend to interfere unduly with management's judgment, as they share the employer's interests in a healthy and prosperous enterprise" (pp. 142 and 143). When assuming, as J. Crispo does, that "the managerial process is certain to become more politicized"' (p. 144) there is no another way for managers but to learn how reconcile the political game with the priorities of business.

Alexander J. MATEJKO

University of Alberta

Une analyse des gains au Canada, par Peter Kuch et Walter Haessel, Ottawa, Statistique Canada, 1979, 272 pp.

Cet ouvrage qui s'inscrit dans la série Etude analytique du recensement constitue une des études les plus fouillées qui ait été publiées sur l'analyse des revenus d'emploi au Canada. Durant la dernière décennie, les études sur la répartition et les disparités de revenu se sont multipliées au Canada. Ces travaux portaient principalement sur le rendement de l'investissement en capital humain, la discrimination selon le sexe et l'influence de caractéristiques personnelles, comme l'appartenance ethnique. Non seulement, le présent ouvrage s'inscrit dans cette lignée, mais par son approche intégrée, il complète les précédentes analyses sur l'investissement en capital humain en y incorporant en particulier des variables de marché. De plus, l'utilisation de variables d'interaction permettant d'estimer le rendement de l'investissement en éducation et en expérience, selon les groupes ethniques, débouche sur d'importantes considérations de politiques économiques.

Les auteurs utilisent l'analyse de régression multiple avec estimation des coefficients selon la méthode du maximum de vraisemblance. La variable dépendante retenue est la moyenne du logarithme des gains annuels ou hebdomadaires ou ce qui revient au même le logarithme de la moyenne géométrique des gains. On sait que de nombreuses études menées sur la répartition des revenus indiquent que les distributions de fréquences des revenus peuvent être assez bien représentées par une fonction de densité lognormale. Quoique l'étude de Lydall citée par les auteurs n'apportent pas de conclusion aussi catégorique. En effet Lydall a testé cette hypothèse pour plus de 30 pays pour conclure que «La partie centrale de la distribution, entre peut-être le dixième et le quatre-vingtième percentile à partir du haut, est près d'être lognormale, mais les extrémités de la distribution contiennent trop de fréquences par rapport à la distribution lognormale». Les auteurs reconnaissent implicitement que cette conclusion quelque peu mitigée peut restreindre la précision de l'estimation des coefficients de la fonction de gains sous l'hypothèse d'une distribution lognormale. Mais, ils croient que les nombreux avantages qu'elle comporte sont suffisants pour justifier son emploi. La distribution lognormale est, en particulier, compatible avec la théorie du capital humain, qui explique le mode de formation des revenus. De plus, sa signification économique est claire, en ce que la variance des logarithmes de gains est une mesure non ambiguë de leur disparité. Enfin son utilisation est facile. On peut ajouter ici que les nombreuses études qui ont été faites pour expliquer la forme mathématique que la répartition des revenus, amènent souvent comme explication à la présence d'une forme lognormale, le fait que les facteurs explicatifs se renforcent eux-mêmes. Or les auteurs ont eux-mêmes observé ce phénomène. Ainsi, il existe une corrélation simple de .87 entre le niveau d'instruction et le taux de rendement.

Quant aux facteurs explicatifs des dispa- 
rités, ils sont regroupés en trois catégories: les caractéristiques personnelles, les effets de marché et enfin les effets de l'investissement en capital humain. Les effets de marché comprennent les facteurs relatifs à la situation géographique, à l'occupation ainsi qu'au secteur d'activité. Des variables d'interaction entre ces deux derniers facteurs et la localisation sont introduites afin de saisir les effets des cédules particulières d'offre et de demande de travail par catégories occupationnelles ou industrielles selon les régions. Quant au capital humain, il se rapporte naturellement au rendement de l'instruction et de l'expérience. Enfin, les caractéristiques personnelles incluent des variables telles que l'état matrimonial, le bilinguisme, la situation salariale ou non, le statut d'immigrant et l'appartenance ethnique.

Parmi les variables énumérées ci-dessus, seule la période d'immigration n'a pas d'influence sur les gains. L'ensemble des variables de marché contribuent de façon significative à la détermination des gains annuels, et d'un autre côté, les caractéristiques personnelles et les effets de l'investissement en capital humain demeurent significatifs après que l'on a pris en compte les variables d'effets de marché, c'est-à-dire, après avoir normalisé pour le secteur d'activité, la profession, la région et le taux moyen de salaire dans la profession. Cette dernière variable étant incluse afin de tenir compte de l'élasticité de l'offre et de la demande régionale selon les professions.

Une combinaison très fertile des variables est celle de l'interaction entre les variables de capital humain et celle de l'appartenance ethnique. Ce jumelage des facteurs permet entre autres d'estimer le rendement sur le capital humain selon l'appartenance ethnique. Les auteurs observent que l'ambitus des taux de rendement est considérable. Ainsi, il varie de $1.38 \%$ chez les indiens autochtones à $7.47 \%$ chez les Canadiens d'origine britannique. C'est d'ailleurs ce facteur de rendement sur le capital humain qui explique la plus grande part des écarts de revenus entre groupe ethnique. Ainsi, comparant les salariés d'origine britannique avec ceux d'origine française, les auteurs observent que le taux de rendement inférieur de ce dernier groupe se traduit par un écart de $23.5 \%$ entre les gains, alors que l'on attribue seulement $4.7 \%$ des écarts de revenus entre ces deux groupes aux différences de niveau d'instruction et d'expérience. L'effet net de l'appartenance ethnique française est estimé à $8.8 \%$.

Les auteurs concluent de cette analyse que ces résultats donnent un ton pessimiste aux politiques économiques. Ainsi, étant donné le faible taux de rendement pour les indiens autochtones, la hausse de leur niveau d'instruction à celui de la moyenne nationale augmenterait leur revenu d'au plus $4 \%$.

Cette analyse d'ailleurs confirme nos connaissances théoriques sur l'asymétrie de la distribution des revenus. Plus les personnes ont un rendement élevé, plus leur niveau de qualification l'est également et plus aussi leurs qualifications sont supérieures et plus leur offre de travail (en terme de quantité) tend à s'élever. Plusieurs auteurs ont, en effet, déjà signalé que le renforcement mutuel des facteurs de disparités constitue une importante explication de l'asymétrie de courbes de distribution des revenus. Les présents résultats confirment ces hypothèses au-delà de toute attente.

Ces premiers résultats ne concernent que le revenu des hommes. Les auteurs consacrent deux chapitres de leurs études aux gains des femmes et aux effets de l'état matrimonial sur la répartition des gains.

Une première observation que les auteurs font à propos de ce dernier groupe, est «que les variations entre les niveaux d'instruction atteints et les rendements réalisés ne sont pas une importante source d'écarts entre les gains des femmes des divers groupes ethniques». En fait, il sembe se produire des effets de compensation entre les taux de rendement et les caractéristiques personnelles. À l'intérieur même des groupes ethniques, les facteurs les plus importants d'explication des inégalités des gains des femmes sont le nombre de semaines travaillées, la durée de l'expé- 
rience et le travail à temps partiel. Le premier de ces facteurs étant de loin le plus important. D'ailleurs, l'influence du nombre de semaines travaillées sur les gains des salariés s'avère assez importante pour que les auteurs y consacrent un chapitre de leur ouvrage.

Pour fin de comparaison des gains entre sexe, les auteurs ont retenu les groupes des hommes non-célibataires et des femmes célibataires, car pour eux, il est clair que ces dernières «sont beaucoup plus semblables aux hommes que les femmes non-célibataires sur le plan du travail accompliin. En fait, leurs caractéristiques personnelles utiles sur le marché du travail des hommes non-célibataires et des femmes célibataires ne sont pas statistiquement distinctes. Pourtant, il existe quand même des écarts de rémunération entre ces deux groupes provenant du fait que le taux de rendement des caractéristiques personnelles est plus élevé et de façon substantielle pour les hommes que pour les femmes.

D'un autre côté, il est intéressant de rappeler les résultats selon lesquels les écarts de gains entre femmes célibataires et non-célibataires dépendent à $80 \%$ de différence dans les caractéristiques personnelles comme le niveau d'instruction ainsi que du nombre de semaines travaillées. Mais le taux de rendement de ces caractéristiques personnelles lui est identique pour les deux groupes. Par contraste, rappelons ici que pour les hommes de catégories homologues, c'est l'inverse qui se produit: l'écart entre les deux groupes du taux de rendement des caractéristiques personnelles constitue la majeure source de disparité de gains entre les hommes célibataires et noncélibataires.

De l'ensemble de cette étude qui analyse un nombre impressionnant de facteurs de disparités de revenus, il est difficile de tirer des conclusions définitives, quoique certaines d'entre elles paraissent bien se dégager. Ainsi, l'éducation en elle-même, c'est-à-dire son niveau, n'est pas suffisante pour éliminer les disparités de revenus. Au contraire, elle semble les accentuer du fait de la présence de corrélation entre les niveaux d'éducation et les taux de rendement. De plus, certains groupes ont un niveau de rendement sur leur capital humain si faible ou tellement plus bas que le groupe de référence que l'amélioration des niveaux devrait se faire à un rythme phénoménal si on voulait rejoindre la moyenne nationale. Sans compter que certains taux de rendement, déjà très bas, risquerait encore de baisser si ces politiques avaient pour effet de créer une offre excédentaire de travail. De plus, le rôle de l'instruction n'a pas pour tous les groupẹs la même importance comme facteur d'accès à certaines activités économiques. Ceci est établi de façon positive pour des femmes, mais est probablement exact aussi pour certains groupes ethniques. Plus que l'offre, il me semble que c'est la demande de travail qui décidera de la persistance ou pas des disparités.

Gérald MARION

\section{Université de Montréal}

\section{Collective Bargaining and Conflict Resolution in Education: The Evolution of Public Policy in Ontario, by Bryan M. Downie, Kingston, Ontario, Industrial Relations Centre, Queen's University, 1978, $179 \mathrm{pp}$.}

A glance at almost any issue of a major Canadian daily newspaper is enough to show that public sector industrial relations in this country is in a state of evolution, to say nothing of occasional chaos. In part because of this friction and unrest, efforts are proceeding on a number of fronts to address the dilemmas inherent in collective bargaining in the public sector, including the problems of right-to-strike, fair compensation, and protection of the public. In this time of change, we badly need detailed and in-depth analyses and evaluation of these efforts in order to formulate new and better public policies.

Among the most useful analyses in recent years have been Arthur's Collective Bargaining by Public Employees in Canada and the work of Woods' Task Force on Labour Relations. Now, in the field of education, Bryan Downie's new book makes an important and significant contribution by ex- 